\title{
Schulleitungshandeln an ausgezeichneten Schulen
}

\author{
Erste Einblicke und empirische Befunde
}

\author{
Michael Schratz • Markus Ammann • Niels Anderegg • \\ Alexander Bergmann • Malte Gregorzewski • Werner Mauersberg • \\ Veronika Möltner
}

Angenommen: 24. Mai 2019 / Online publiziert: 21. Juni 2019

(C) Der/die Autor(en) 2019

Zusammenfassung Die Autonomisierung im Schulwesen hat die Aufgaben und das Handlungsfeld von Schulleitungen in den letzten Jahren verändert. Einerseits ist für sie das gezielte Wahrnehmen von Führung notwendig geworden, andererseits erfordert die Qualitätssorge über die Rechenschaftspflicht verstärkte Aufmerksamkeit. Im Zuge dieser Entwicklung setzt sich die Schulleitungsforschung stärker mit der Frage nach dem Einfluss von Führung auf den Unterricht und dessen Ergebnisse auseinander. Im vorgestellten Forschungsprojekt wird das Handeln von Schulleitungen an lernwirksamen Schulen in Deutschland untersucht, deren Erfolge über die sechs Qualitätsbereiche des Deutschen Schulpreises evaluiert worden sind. Über einen mehrperspektivischen Zugang qualitativer Forschungsmethoden wurde in mehrtägigen Feldphasen erkundet, wie sich Schulleitungshandeln im alltäglichen Schulgeschehen zeigt. Der phänomenologische Zugang bringt als Antwort ein vielschichtiges Geflecht von Facetten zutage, die in Form eines Registers zusammengefasst werden. An einer Beispielfacette wird aufgezeigt, wie sich deren Eigenschaften in unterschiedlichen Ausprägungen konkretisieren. Die Untersuchung möchte einen Beitrag zur Frage nach erfolgreichem Führungshandeln im deutschsprachigen Raum leisten und jene Aspekte der Schulentwicklung aufgreifen, die für die Qualifizierung und Professionalisierung von schulischen Führungspersonen im deutschen Sprachraum und darüber hinaus bedeutsam sind.

\footnotetext{
M. Schratz $(\bowtie) \cdot$ M. Ammann · A. Bergmann · M. Gregorzewski · W. Mauersberg · V. Möltner Institut für LehrerInnenbildung und Schulforschung, Universität Innsbruck,

Fürstenweg 176, 6020 Innsbruck, Österreich

E-Mail: Michael.Schratz@uibk.ac.at

N. Anderegg

Pädagogische Hochschule Zürich, Lagerstraße 2, 8090 Zürich, Schweiz

V. Möltner

Pädagogische Hochschule Tirol, Pastorstraße 7, 6010 Innsbruck, Österreich
} 
Schlüsselwörter Schulleitung · Schul- und Unterrichtsqualität · Well-being · Exzellente Schulen

\section{Leadership for learning in excellent schools}

First insights and empirical findings

Abstract The recent movement towards more autonomous school systems has expanded the field of action of principals and transformed the scope of their duties. Accordingly, the focus of research on school leadership has also become more devoted to the question to which extent principals can have an influence on school quality. The study explores the relationship between school leadership and the effects of teaching and learning in schools which were awarded the German School Prize according to its comprehensive framework referring to six quality areas. The phenomenologically oriented approach tries to find answers to the research question how leadership shows in school.

During the three days of fieldwork principals, teachers and students were interviewed according to the effects of principals' acts. The answers from the individual findings were compiled as a response register that shows the actions of school leaders in a multitude of diverse facets of leadership. A sample facet from the research work is presented to illuminate the general concept of the theoretical facet model of school leadership. The findings of the research project aim at contributing to professional learning in school leadership and school improvement in German speaking countries and beyond.

Keywords School Leadership · Leadership for Learning · Well-being · Excellent Schools

\section{Problemaufriss}

Die weltweit im Bildungswesen zu beobachtende Zunahme von Autonomisierung (Salokangas und Ainscow 2018) erhöhen die am jeweiligen Schulstandort eigenverantwortlich zu treffenden Entscheidungen. Damit haben sich in den letzten Jahren auch die Verantwortlichkeiten verschoben und zu einer Stärkung der Rolle von Schulleitung ${ }^{1}$ geführt (Schratz und Hartmann 2009). Die damit verbundene Neuausrichtung der Schulgovernance im Hinblick auf eine ergebnisorientierte Steuerung (Altrichter und Maag Merki 2010; Kohlstock und Heinrich 2015) hat neue Anforderungen an Führungspersonen im Bildungswesen gestellt. Diese müssen am jeweiligen Standort eine Balance zwischen festgelegten rechtlichen Vorgaben und der eigenverantwortlich pädagogischen Gestaltung der Schule im jeweiligen schuli-

\footnotetext{
1 In dieser Studie werden die Begriffe Schulleiter/in bzw. Schulleitung ohne präzise Differenzierung der betreffenden Akteure verwendet, da die hier untersuchten Steuerungsprozesse in ein responsives Geschehen eingebunden sind und Schulleitung sich nicht immer auf eine einzelne Person beziehen kann. Dies wird auch im Titel des Beitrags signalisiert.
} 
schen Kontext finden, um den komplexen, zum Teil neuen Ansprüchen gerecht zu werden.

Im Spannungsfeld zwischen den komplexen Anforderungen externer Rechenschaftspflicht im Reformgeist einer neuen Steuerungsdynamik sind pädagogische Führungspersonen gefordert, Prozesse der Schulentwicklung zu initiieren und die damit verbundenen vielschichtigen Interaktionsgeflechte zu leiten und zu steuern. Schulleitungen zeigen sich daher zunehmend mit Fragen der Etablierung eines evidenzverpflichteten Umgangs mit Ergebnissen aus Lernstandserhebungen, Vergleichsarbeiten u. ä. konfrontiert (vgl. Schratz et al. 2019). Im Zuge dessen hat sich die Aufmerksamkeit der Schulwirksamkeitsforschung auf die Steigerung von Schülerleistungen verlagert, weshalb das Forschungsinteresse aktueller Studien darin liegt, inwiefern Schulleiter/innen Einfluss auf das Lernen von Schüler/innen haben.

Der Zusammenhang zwischen Schulleitung und dem Lernen der Schüler/innen, bislang auch als „black hole“ (Leithwood et al. 2009) oder „,black box“ (Heck und Hallinger 2010) bezeichnet, wird zunehmend mehr und methodisch aus verschiedenen Perspektiven erforscht. Die vorliegenden Befunde deuten zusammenfassend in die Richtung, dass Schulleitung sowohl indirekt als auch direkt Einfluss auf das Lernen von Schüler/innen nimmt (vgl. Bell et al. 2003; Hallinger und Heck 2011; Leithwood et al. 2004, 2009; Townsend und MacBeath 2011a, 2011b; Pietsch et al. 2016).

Allerdings gibt es wenig Hinweise darauf, welches Schulleitungshandeln tatsächlich zum Erfolg führt und das Lernen der Schüler/innen positiv beeinflusst. Darüber hinaus stellt sich die Frage, wieweit die Ergebnisse von Studien aus dem englischsprachigen Raum auf Grund der Unterschiede in der angelsächsischen Tradition und Kultur der Schulen auf die deutschsprachigen Bildungssysteme übertragbar sind, da sich die Professionsverständnisse der Lehrer/innen der betreffenden Kulturen und der Bildungsaufträge der jeweiligen Länder unterscheiden (vgl. Shirley 2017; Klein 2016).

Das Forschungsprojekt „Schulleitungshandeln an den Preisträgerschulen des Deutschen Schulpreises“2 ${ }^{\text {"2 }}$ geht der Frage nach, wie sich Schulleitungshandeln an lernwirksamen Schulen in Deutschland zeigt. ${ }^{3}$ Neben den Leistungen der Schüler/ innen beinhaltet der Begriff der Lernwirksamkeit einer Schule weitere Aspekte und schließt ein breiteres Verständnis von Lernen und Bildung mit ein, das auch den Qualitätsbereichen des Deutschen Schulpreises zugrunde liegt (vgl. Beutel et al. 2016). Die Perspektive, mit der dabei schulischer Erfolg verstanden wird, lässt sich in den im Laufe der letzten Jahre im internationalen Policy-Diskurs angedeuteten Themenwechsel hin zum schulischen „Well-being“ einordnen (Ecclestone 2007; OECD 2017; Spratt 2019).

\footnotetext{
2 Dieses Forschungsprojekt wurde von der Robert Bosch Stiftung gefördert.

3 Der Deutsche Schulpreis wurde von der Robert Bosch Stiftung und der Heidehof Stiftung initiiert und wird in Kooperation mit den Medienpartnern ARD und DIE ZEIT umgesetzt. Mit dem Deutschen Schulpreis wollen dessen Initiator/innen die Leistungen der ausgezeichneten Schulen öffentlich würdigen und deren Innovationskraft für die Schulentwicklung in die Fläche nutzbar machen, denn Schulwettbewerben wird ,sowohl eine emotionale Komponente (Hoffnung auf Anerkennung, Ehrung) zugesprochen als auch eine funktionale Komponente (Nutzen für Schulentwicklung)“ (Albers 2016, S. 9).
} 
In diesem Beitrag folgt zunächst eine weiterführende theoretische Verortung in den Diskurs des „Well-being“. Im Anschluss daran wird das methodische Vorgehen entfaltet. Untersucht wurden alle Schulen, die einen der ausgelobten Preise gewonnen haben und an denen im Untersuchungszeitraum dieselbe Schulleitung im Amt war wie zum Zeitpunkt der Auszeichnung. In weiterer Folge wird exemplarisch eine theoretisch erarbeitete Kategorie aus den bisher gewonnen Daten dargestellt, welche den zugrundeliegenden Forschungsansatz repräsentiert. Zwischenzeitlich konnten 55 derartige Kategorien identifiziert werden. Diese können beispielhaft als mögliche Antworten auf die Frage verstanden werden, wie sich Schulleitungshandeln an lernwirksamen Schulen zeigt. Der Beitrag endet mit einem zusammenfassenden Ausblick und einer ersten Einordung der gewonnenen Kategorien in ein Facettenmodell von Schulleitung.

\section{Theoretische Positionierung}

Da das Forschungsinteresse auf Schulleitungshandeln an lernwirksamen Schulen abzielt, gilt es zunächst, das dem Projekt zu Grunde liegende Verständnis von Lernen im Hinblick auf Lernwirksamkeit zu explizieren. In zahlreichen Beiträgen, die dieser Frage nachgehen, wird school effectiveness über Leistungsergebnisse aus standardisierten Tests definiert. Heck und Hallinger (2010, S. 14) bezeichnen Lernen in ihrem Beitrag beispielsweise als ,the growth in student learning in math scores of longitudinal student cohort during the study". Eine derart formulierte Orientierung an standardisierten Leistungsmessungen fachlichen Unterrichts vernachlässigt allerdings für Schule und Unterricht nicht unbedeutendes Handlungsgeschehen und reduziert die Komplexität und Vielfalt schulischen Lernens auf Output (vgl. Cappella et al. 2016). Biesta sieht darin die Gefahr des Verlustes des Pädagogischen, wenn er argumentiert „,if we take the risk out of education, there is a real chance that we take out education altogether" (Biesta 2013, S. 1). Durch ihre Anlage konzentrieren Leistungsvergleiche nach Messner (2003, S. 409) ,die Bildungsfrage notwendig auf das "Produkt“ schulischen Lernens im Sinne messbarer Leistungen. Die Prozesse des Sich-Bildens, das gesamte Handlungsgefüge schulischen Unterrichts, werden demgegenüber, wenigstens zunächst, sekundär." Biesta (2017) argumentiert, dass in einem solchen Verständnis Schüler/innen als Objekte eines sicher herstellbaren Lernprozesses und nicht mehr als Subjekte einer bildenden Aneignung angesehen werden. Prozesse des Sich-Bildens sind aber für ein erweitertes Verständnis von Lernen und somit auch qualitätsvoller Schule unabdingbar. Dies wird etwa auch in verschiedenen Qualitätsmodellen - in Österreich bspw. SQA-Allgemeinbildung (vgl. SQA-online 2019) über Qualitätsbereiche (Lernerfahrung und Lernergebnisse, Lernen und Lehren, Lebensraum Klasse und Schule, Führung und Schulmanagement, Professionalität und Schulentwicklung sowie Schulpartnerschaft und Außenbeziehungen) zum Ausdruck gebracht (Altrichter et al. 2012). Ein derartig entfaltetes Verständnis stellt unserer Meinung nach die Orientierungspunkte für gelungene schulische Lernprozesse zur Verfügung, welche ihr Ziel in bildenden Lernerfahrungen von Schülerinnen und Schülern haben. 
Shirley (2016) ortet im Deutschen Schulpreis eine ,gesellschaftliche Wiederbelebung von Schule nach einer anderen und neuen Logik“ (S. 9), die er gemeinsam mit Hargreaves (Hargreaves und Shirley 2009; 2012) als „Fourth Way“ beschreibt. Im Hinblick auf eine langfristige Perspektive von Bildung sehen sie diesen vierten Weg als Wendepunkt in der Bildungsreform, der die Art und Weise, wie wir Lernen verstehen und Schule gestalten, auf Jahre beeinflussen wird. So kündigt sich in den letzten Jahren im internationalen Policy-Diskurs ein Themenwechsel an, der ,Well-being“ zum Fokus bildungspolitischer Aufmerksamkeit macht (Ecclestone 2007) und sich auch in der OECD Studie „How's Life?“ spiegelt (OECD 2017). Ausgelöst wurde diese Neuorientierung unter anderem durch die zunehmende Verunsicherung junger Menschen im Hinblick auf ihre Lebens- und Arbeitsperspektiven aufgrund ökonomischer, sozialer und psychischer Einflüsse in der gesellschaftlichen Entwicklung (Damon 2009; Helliwell et al. 2016). Die kanadische Provinz Ontario hatte bereits 2009 ein Gesetz verabschiedet, das jede regionale Schulbehörde verpflichtet, , Wellbeing “ als Verantwortung an jede Schule zu übertragen (Ontario 2016).

Unter der politischen Perspektive von „Well-being“ ist nicht nur das persönliche Wohlbefinden einzelner Personen (Schüler/innen, Lehrpersonen usw.) gemeint, sondern auch das ,organisationale Well-being“, das ein förderliches Umfeld und eine tragfähige Kultur zur Erreichung der damit verbundenen Ziele nahelegt (ebenda). In dieser Hinsicht nehmen erfolgreiche Schulen ihre Verantwortung für Kinder und Jugendliche nicht segmentär, sondern ganzheitlich wahr und verantworten sich aktiv gegenüber den Beteiligten und der Öffentlichkeit. Sie ,,erfassen Lernen und Leistung aus mehreren Perspektiven und berücksichtigen dabei verschiedene Bezugskriterien ebenso wie die Blickwinkel und Urteile der Beteiligten - der Schülerinnen und Schüler selbst, ihrer Eltern und der Lehrerinnen und Lehrer. Sie schaffen Gelegenheiten, bei denen Kinder ihre Talente und Interessen entwickeln können." (Fauser et al. 2007, S. 10).

Zusammenfassend ist die Wahrnehmung einer derart vielperspektivischen Orientierung für Schulleitungen eine große Herausforderung. Dabei spielt auch der Systemblick eine wichtige Rolle. Lernen, das nachhaltig für das weitere Leben wirken soll, erfordert das Zusammenspiel unterschiedlicher Faktoren - nicht nur im Unterricht selbst, sondern an der Schule als Ganzes und darüber hinaus. Schulleitungen gelingt es sehr unterschiedlich, mit den Anforderungen einer im stärkeren Maße eigenverantwortlichen Schule umzugehen (Harazd 2010) und die Chancen der pädagogischen Gestaltung wahrzunehmen. Daher liegt dieser Studie ein Verständnis von lernwirksamen Schulen zugrunde, das in diesem spannungsreichen Zusammenspiel zwischen Schul-, System- und gesellschaftlicher Entwicklung für die Erforschung von Schulleitungshandeln bedeutsam ist.

\section{Methodologischer und methodischer Zugang}

Die leitende Fragestellung der Untersuchung orientiert sich entlang der Forschungsfrage, wie sich Schulleitungshandeln an erfolgreichen Schulen zeigt. Dieses ,Sichzeigen“ verweist auf ein erkenntnisgenerierendes Verfahren, das zunächst Phänomene im Handlungsfeld von Schulleitung erkunden und ,zu den Sachen selbst“ 
vordringen möchte. Dazu ist eine ,phänomenologische Forschungsgesinnung“ (Gadamer 1963/64) dienlich, die eine Offenheit in den Zugängen zu den Phänomenen ermöglicht, mit denen die Forschenden konfrontiert werden. Die so skizzierte ,Forschungsgesinnung' zeigt sich unter anderem in der bereits von Husserl (1962) skizzierten Epoché, welche das Einklammern der bereits vorhandenen theoretischen Konzepte erfordert, damit diese nicht von vornherein an den Forschungsgenstand herangetragen werden. „Damit ist jene Grundorientierung des Forschungsinteresses gemeint, zu den Sachen selbst durchzustoßen“ (Kron 1999, S. 203). Sie führt die Forschenden ,,Zu der persönlichen Erfahrung der eigenen Erkenntnisfähigkeit und -leistung“ (ebd.) - im Sinne von Husserls Argumentation „Natürliche Erkenntnis hebt an mit der Erfahrung und verbleibt in der Erfahrung“ (Husserl 1990, S. 98).

Das phänomenologische Grundverständnis, welches dem Beitrag zu Grunde liegt, spürt den Erfahrungen, welche die verschiedenen Akteure (Schulleitungen, Lehrkräfte und Schülerinnen und Schüler) in Schulen haben, nach. Es versteht diese als Erfahrungen des Führens und Geführtwerdens (Ammann 2018). „Phenomenology as a philosophy of experience means the attempt to understand the experiences of the world, the other and of myself, even if there is an inevitable distance between my concrete, situated experiences and my return to them while I am talking or thinking about them." (Meyer-Drawe 2017, S. 17) Die Forschungsfrage wird demnach nicht über vorgegebene Indikatoren zu beantworten versucht, sondern über induktiv erarbeitete Kategorien. In diesen Kategorien werden die verschiedenen Erfahrungen des Führens und Geführtwerdens zunächst in den einzelnen Schulen und dann auch schulübergreifend verdichtet. Sie werden dadurch generiert, dass die im Feld gewonnenen Daten zunächst als eine Art „Antwortregister“ (Waldenfels 2007) über das Sammeln von Ergänzungen des Satzbeginns „,Schulleitungshandeln an lernwirksamen Schulen zeigt sich als ...“ zusammengestellt wurden. Die so gewählte Formulierung ist phänomenologisch inspiriert und orientiert sich konsequent an dem, was sich den Forscher/innen über das Alltagshandeln von Schulleitungen im Feld zeigt. Es kann gewissermaßen als Spurensuche nach den grundlegenden Tätigkeiten und deren Auswirkungen im jeweiligen Schulkontext gedeutet werden.

Zur Gewinnung von diesbezüglichen Forschungsdaten fanden an insgesamt 28 Preisträgerschulen des Deutschen Schulpreises jeweils dreitägige Feldphasen an den Schulen selbst statt. Alle in der Studie erfassten Schulen haben eine dreistufige Bewertung auf Basis der Qualitätserfordernisse des Deutschen Schulpreises durchlaufen, ehe sie in diese Untersuchung aufgenommen und in der Feldphase besucht wurden. Den ausgezeichneten Schulen, die als idealtypische Fälle charakterisiert werden können (Lamnek 2005, S. 314), wurde in allen sechs Qualitätsbereichen Leistung, Umgang mit Vielfalt, Unterrichtsqualität, Verantwortung, Schulklima, Schulleben und außerschulische Partner und Schule als lernende Institution (vgl. Beutel et al. 2016) durch eine unabhängige Jury hohe Qualität zugesprochen. Dem Juryurteil zufolge gelingt es den Akteuren an diesen Schulen im Hinblick auf die genannten Qualitätsbereiche eine hohe Wirkung zu erzielen, was auch folgendes Zitat nahelegt:

Solche Schulen schaffen es, bei unterschiedlichen Rahmenbedingungen Kinder und Jugendliche für Leistung und Kreativität zu begeistern, ihre Lernfreude und 
ihren Lebensmut zu stärken und sie zu Fairness und Verantwortung zu erziehen. Es gelingt ihnen, mit Lehrerinnen und Lehrern, Schülerinnen und Schülern und Eltern angesichts der enormen Veränderungen in der Gesellschaft, in der Kultur und in den alltäglichen Lebensverhältnissen sich immer wieder neue Ziele zu setzen und diese auch zu erreichen (Schratz 2017, S. 231).

Die 28 untersuchten Schulen konnten nachweislich in allen Bereichen (auch im Bereich der Leistung ${ }^{4}$ ) zeigen, dass sie sowohl auf einer Mesoebene als auch auf einer individuellen Mikroebene in der Lage sind, hohe Qualitätserwartungen innerhalb eines breiten Verständnisses von Schulbildung zu erfüllen. Es sind Schulen, die alle unter verschiedensten Rahmenbedingungen Schulentwicklungsprozesse initiiert haben, die zu ausgezeichneten Ergebnissen beigetragen haben. Dabei handelt es sich weder ausschließlich um Schulen in herausfordernden Lagen, noch um solche, die kontextuell besonders begünstigt sind. Im Rahmen dieses Forschungsprojekts werden die Schulen zunächst als Fallstudien untersucht; in der Folge werden übergeordnete Erkenntnisse generiert, wobei dabei auch theorienprüfend und in weiterer Konsequenz theorieergänzend bzw. -ändernd vorgegangen werden kann (vgl. Oswald 2003, S. 81). „Der Einzelfall interessiert nur, wenn er auf etwas Allgemeineres verweist, wenn seine Interpretation zu Erkenntnissen führt, die über ihn hinausreichen“ (ebenda, S. 73). Fallstudien als solche sind keine vorab definierten Untersuchungsmethoden, sondern vielmehr eine spezifische Art des Zugangs zum Feld (vgl. Lamnek 2005, S. 298, 311).

Das Forschungsprojekt versteht sich in der Tradition der qualitativen, interpretativen Sozialforschung (Lamnek 2005; Rosenthal 2014). Für die Datenerhebung erfolgte zunächst eine umfangreiche Dokumentenanalyse (Bewerbungsunterlagen der Schulen, Beurteilungsdaten aus Jurybewertungen, evidenzbezogene Berichte der Juryteams über die Schulbesuche, Nominierungsbewertungen durch die Jury nach den sechs Qualitätsbereichen, Urteile zur Auslobung der Preisträger). Die Aufenthalte im Feld wurden in Form der teilnehmenden Beobachtung (Schulleitung, Unterricht und Schulleben) mit der Erstellung von Feldnotizen (vgl. Dellwing und Pruse 2012, S. 147ff; Reh 2012) und Vignetten (vgl. Schratz et al. 2012) durchgeführt. Weiters kamen Fokusgruppengespräche (vgl. Wolff 2005, S. 511; Lamnek 2005, S. 317, 547 ff.) mit Lehrkräften und Schüler/innen sowie Interviews mit der Schulleitung (halbstandardisiert am ersten, offen am dritten Feldtag; vgl. Kruse 2014) zum Einsatz. Schulspezifische Dokumente aus den Feldaufenthalten wurden bei der Auswertung berücksichtigt. Die Feldphasen an den einzelnen Schulen dauerten jeweils drei Tage, die Datenbasis bilden in Summe (Einzelpersonen und Gruppen) 112 Gespräche.

Mit Hilfe des gewählten qualitativ-empirischen Forschungsdesigns soll ein mehrperspektivisches Bild auf das Schulleitungshandeln an den einzelnen Schulen erschlossen werden (vgl. Lamnek, 2005, S. 298), wozu in den Fallstudien versucht wird, über Triangulationsprozesse unterschiedliche Aspekte, und Dimensionen zu

\footnotetext{
${ }^{4}$ Hierzu verweisen die Schulen u. a. auf vorliegende zentrale und/oder landesweite Vergleichsdaten wie beispielsweise Ergebnisse der zentralen Vergleichsarbeiten (VERA-3 und VERA-8), Übertrittsquoten ins Gymnasium, Abiturprüfungsleistungen, usw.
} 
erfassen (ebenda, S. 299). ,Triangulation beinhaltet die Einnahme unterschiedlicher Perspektiven auf einen untersuchten Gegenstand oder allgemeiner: bei der Beantwortung von Forschungsfragen. Diese Perspektiven können in unterschiedlichen Methoden, die angewandt werden, und/oder unterschiedlichen gewählten theoretischen Zugängen konkretisiert werden, wobei beides wiederum mit einander in Zusammenhang steht bzw. verknüpft werden sollte. Weiterhin bezieht sie sich auf die Kombination unterschiedlicher Datensorten jeweils vor dem Hintergrund der auf die Daten jeweils eingenommenen theoretischen Perspektiven." (Flick 2008, S. 10).

Das Projekt zeichnet sich somit sowohl durch eine Investigatoren-Triangulation (intersubjektive kommunikative Validierung der Datenauswertung innerhalb des Forschungsteams) sowie eine methodologische Triangulation (unterschiedliche Forschungsmethoden) aus. Mit dem Ziel, verschiedene Perspektiven auf das Forschungsfeld zu eröffnen, wurde die Dokumentenanalyse selbstständig und isoliert von den qualitativ im Feld gewonnenen Daten vollzogen. Die Ergebnisse wurden nach Abschluss der gesamten Auswertung miteinander abgeglichen und zusammengeführt. Darüber hinaus wurden sowohl für die Dokumentenanalyse als auch zur Auswertung und kommunikativen Validierung der Daten weitere Mitglieder in die Forschungsgruppe aufgenommen, die nicht im Feld aktiv selbst Daten erhoben hatten und somit einen nicht vom Feld geprägten Blick auf die Daten richten konnten.

Der Definition von Flick folgend, wurden die Effekte der Triangulation der Auswertung der qualitativ gewonnenen Daten in einem zirkulären Stufenprozess wiederkehrend kontrolliert. Die Auswertung der in den Gesprächen gewonnenen und aufgezeichneten Daten erfolgte unter Zuhilfenahme der Software ATLAS.ti, welche die Möglichkeit bot, sowohl induktiv als auch deduktiv zu codieren. Die Datenauswertung erfolgte, orientiert an der Grounded Theory (Strauss und Corbin 1996), nach einem fünfstufigen Verfahren, das ,,die Forschungsobjekte ebenso wie die zwischen ihnen bestehenden Relationen in diesem Prozess konstruiert" (Strübing 2008, S. 16). Diese Orientierung hat sich für die Ausformulierung von Kategorien, die sich in einem phänomenologischen Sinne nah an den Erfahrungen der Akteure im Feld orientiert, als Regelwerk gut bewährt. Auf der Grundlage eines gemeinsam erstellten Codebuchs wurden alle Gespräche zunächst offen codiert und zu für die jeweilige Schule passenden Kategorien verdichtet. Die entlang eines entwickelten Rasters ausformulierten Kategorien wurden in der Folge schulübergreifend in Kategoriengruppen geclustert, die inhaltlich auf gleichen bzw. semantisch ähnlichen Codes beruhen. Die so identifizierten Codes wiederum wurden in einem vierten Schritt axial codiert, was darauf abzielte, mögliche Zusammenhänge zwischen den Codes herauszuarbeiten, um in einem fünften Schritt, die schulübergreifenden theoretischen Kategorien final auszuformulieren.

Jeder so vollzogene Schritt wurde im Rahmen der Forschungsgruppe kommunikativ validiert und hinsichtlich des Regelbuchs geprüft sowie im Anschluss überarbeitet. Die so entstandenen 55 Kategorien wurden in einem letzten Schritt nochmals entlang der Vorgaben kommunikativ in zumindest einem Sechs-Augen-Prinzip innerhalb der Forschungsgruppe kommunikativ validiert. Jede der so formulierten Kategorien musste dabei bestimmte Eigenschaften erfüllen, die verschiedene Abstraktionsebenen abbilden, sich aber alle stringent ins empirische Material zurückführen lassen. Die Eigenschaften einer Kategorie waren dabei die folgenden: Titel, 
Kurzbeschreibung, Merkmalsausprägungen, Beschreibungen der Merkmalsausprägungen, empirische Belege. Die so formulierten Eigenschaften ermöglichen es, von jeder Abstraktionsebene aus direkt zurück bis zu den einzelnen Interviews zu gehen.

Die im Rahmen der Auswertung der Daten erarbeiteten Kategorien wurden im Sinne des zu erstellenden Antwortregisters (Waldenfels 2007) als Ergänzung des Satzanfangs „Schulleitungshandeln an lernwirksamen Schulen zeigt sich als ..." formuliert. Die so gewonnenen Antworten sind Ausdruck verschiedener und zahlreicher Handlungen von Schulleitungen an den Preisträgerschulen des Deutschen Schulpreises, die nicht isoliert und ohne den schulspezifischen Kontext betrachtet werden können. Vielmehr lassen sich die verschiedenen gewonnenen Kategorien in ihrer Pluralität als Facetten von Schulleitungshandeln betrachten. Der Begriff der Facette stammt aus dem Französischen und hat zwei Bedeutungen. Einerseits werden die beim Schleifen eines Diamanten oder von Glas entstehenden eckigen Flächen Facetten genannt. Andererseits wird der Begriff Facette auch für Teilaspekte eines größeren Ganzen verstanden (Duden 2001, S. 300). Im Wort Facette kommt zum Ausdruck, dass es jeweils nur ein einzelner Einblick in das Handeln von Schulleitungen ist und daneben auch andere Facetten für das Gesamtbild relevant sind. Metaphorisch (Lakoff und Johnson 2001) können diese Facetten mit jenen eines Diamanten verglichen werden. Die Metapher eines geschliffenen Diamanten steht gewissermaßen bildlich für das Zusammenwirken der verschiedenen Facetten im täglichen Fluss von Schulleitungshandeln. Die jeweilige Facette an sich ist eine analytische Trennung einzelner Handlungen von anderen, ohne dabei auf die anderen Facetten zu vergessen und sich derer zu entledigen. Erst das Zusammenspiel der einzelnen Facetten kürt den Rohdiamant zum Brillant und macht ihn einzigartig. Abhängig von Distanz und Nähe zum betrachteten Diamanten werden einzelne Facetten deutlicher und andere verschwinden in der Betrachtung, sind aber dennoch relevant für das Gesamtbild. Dieser Metapher folgend, bezeichnen wir unsere Kategorien als Facetten von Schulleitungshandeln, welche als Teil des Facettenmodells von Schulleitungshandeln verstanden werden können. Eine derartige, nach dem beschriebenen Verfahren induktiv gewonnene Facette wird im nächsten Abschnitt zunächst theoretisch eingeordnet und darauf hinsichtlich der Tätigkeitsbeschreibungen und daraus resultierenden Handlungen von Schulleitungen diskutiert.

\section{Erste empirische Erkenntnisse: Schulleitungshandeln zeigt sich als „... nah dran sein"6}

„Nah dran sein“ ist eine von vielen im Forschungsprojekt identifizierte Facette. Sie antwortet im Sinne des Antwortregisters auf den Satzanfang ,Schulleitungshandeln zeigt sich als ...“. Im Folgenden soll diese Facette exemplarisch vorgestellt, diskutiert und in den Zusammenhang gesetzt werden.

Das Spannungsfeld zwischen Nähe und Distanz spielt im menschlichen Miteinander im Allgemeinen und in der Pädagogik im Speziellen eine wichtige Rolle, da sich darin Beziehungen und Verhältnisse auswirken. Helsper $(2002,2004)$ verweist in seinen Professionsantinomien des Lehrerhandelns auf das Spannungsverhältnis von Nähe und Distanz und arbeitete dessen Bedeutsamkeit für pädagogisches Han- 
Tab. 1 Beispiel für eine Auswertungsfacette (,,.. nah dran sein“) für das Sich-zeigen von Schulleitungshandeln mit jeweiligen Eigenschaften und Ausprägungen

Schulleitungshandeln an lernwirksamen Schulen zeigt sich als ...

„... nah dran sein“"

Eigenschaften Ausprägung

... am Schulumfeld und den

Schulpartnern

Bewusstsein über den Schulkontext (Eltern, Kommune, ...) und die Gestaltungsmöglichkeiten.

Gestalten und Pflege eines Netzwerks und dessen Einbindung (Vereine, Unternehmen, Universitäten) in Schulentwicklung

... an den Personen

Achtsames Ausbalancieren des Spannungsfelds zwischen rechtlichen, wirtschaftlichen und pädagogischen Überlegungen

... an der Forschung

Beschäftigung mit aktuellen wissenschaftlichen Erkenntnissen und Einbindung dieser zur Fundierung und Argumentation des pädagogischen Konzepts der Schule

... am pädagogischen Gesamt- Klares Bewusstsein über Details des Konzeptes und darüber, wie konzept sich Änderungen im Detail auf das Große und Ganze auswirken

deln heraus. Es ist insbesondere auch für das Führungsverhalten bedeutsam, wie Personen in Entwicklungsdynamiken und mikropolitischen Prozessen ihre Beziehungen organisieren. Brüchle et al. (2015) etwa gehen in ihrem Beitrag zur Frage von mikropolitischen Einflusstaktiken in Führungsbeziehungen der Frage nach, welche Strategien junge Führungskräfte in erster Führungsverantwortung wählen, um das Distanz-Nähe Dilemma zu überwinden. Sie identifizieren drei verschiedene Taktiken: der bewusste Einbezug des Teams in Entscheidungsprozesse, inspirierende Appelle sowie Legitimation durch den Verweis auf die Unternehmenspolitik bzw. die eigene Hierarchieposition. Die so formulierten Taktiken stehen exemplarisch für den Versuch des absichtsvollen Ausbalancierens von Nähe und Distanz zu den einzelnen Mitarbeiter/innen, um die eigenen Ziele besser erreichen zu können.

Die in diesem Beitrag entfaltete Facette geht explizit nicht von einem Nähe-Distanz-Dilemma aus, sondern bringt vielmehr ein bewusstes „Nah dran sein“-wollen zum Ausdruck und verkürzt dieses Phänomen zusammenfassend nicht zu einem oszillierenden Spiel zwischen Nähe und Ferne. Das „Nah dran sein“ von Schulleitungen hat sich in zahlreichen Aussagen gezeigt, in denen es um eine besondere Nahbeziehung zu Personen, Gruppen, Institutionen, Gegenständen und Sachverhalten ging. Diese Facette lässt sich an den untersuchten Schulen nicht nur im Umgang mit den Schüler/innen und Lehrer/innen beobachten, sondern zeigt sich auch in einem umfassender gedachten Sinn, wie beispielsweise der Nähe zum Schulumfeld, zur Forschung, zur Organisation und Verwaltung oder zu pädagogischen Inhalten.

Tab. 1 stellt die Facette überblicksmäßig dar, wobei diese in insgesamt vier verschiedenen Eigenschaften ausdifferenziert werden kann. Diese Form der Kategorisierung lehnt sich an die von Strauss gewählte Beschreibung der Kategorie ,Beobachten' an (Strauss und Corbin 1996). Die verschiedenen Eigenschaften werden über eine kurze Beschreibung der jeweiligen Ausprägungen einer detaillierteren und vertiefenden Beschreibung zugeführt. Die Trennung in die verschiedenen Eigenschaften erfolgt an dieser Stelle ausschließlich aus analytischen Gründen, da sich diese, wie beschrieben, teilweise überschneiden bzw. in gegenseitigen Beeinflussungsverhältnissen stehen. 
In weiterer Folge werden die verschiedenen Eigenschaften der Facette unter Einbezug von Originalbelegen aus dem Datenmaterial näher beschrieben und hinsichtlich ihrer erfolgreichen Arbeit auf das diesem Beitrag zu Grunde liegende Verständnis von lernwirksamer Schule befragt. Demnach zeigt sich Schulleitungshandeln als ,nah dran sein“ ...

\section{1 ... am Schulumfeld und den Schulpartnern}

In der Nähe zum Schulumfeld und den Schulpartnern zeigt sich ein wesentlicher Grundbaustein von lernwirksamer Schulleitung. Diese Eigenschaft der Facette deckt sich mit Erkenntnissen der Literaturrecherche (Day et al. 2010; Leithwood und Seashore Louis 2012; Hallinger 2009; Schwarz und Brauckmann 2015; Wiesner et al. 2016). Demzufolge zeichnet sich erfolgreiches Schulleitungshandeln dadurch aus, dass es kontextbezogen bzw. -sensitiv ist, wenngleich sich bestimmte Strategien, Prinzipien und Praktiken immer wieder zeigen. Diese werden allerdings - und das erscheint von besonderer Bedeutung - auf den jeweiligen Kontext bzw. die jeweilige Situation ,,angepasst“.

In Bezug auf kontextbezogenes Schulleitungshandeln spielt die Nähe zum Schulumfeld eine entscheidende Rolle, denn diese ermöglicht es den Schulleitungen die Bedürfnisse, Potentiale und Herausforderungen des Schulumfeldes wahrzunehmen und es in weiterer Folge einzubinden. So entstehen beispielweise Kooperationen mit Jugend- und Sozialeinrichtungen, Unternehmen, Verbänden, Wissenschaftseintrichtungen, NGOs ${ }^{5}$, anderen Schulen oder politischen Vertretungen. Aber auch freiwillige Helfer/innen werden gewonnen, die Zusammenarbeit mit Eltern und Ehemaligen wird forciert oder unterschiedliche Expertisen werden im Unterricht eingebunden. Das „Nah dran sein“ zeigt sich dabei durch das Herstellen und Pflegen eines umfassenden Netzwerkes, in das die Schüler/innen und Lehrpersonen eingebunden werden. Dadurch können fruchtbare Prozesse des Zusammenarbeitens entstehen, von denen sowohl die Schule als auch das Schulumfeld profitiert. Im Folgenden Beispiel werden verschiedene Ebenen dieser Dimension des „Nah dran seins“ deutlich:

Mit der Durchführung des Tanzprojektes (,Elementartanz“) an unserer Schule haben wir in diesem Bereich tiefgreifende Erfahrungen machen können. Die gesamte Schule, SeniorInnen aus Pflegeheimen, das Städtische Orchester, Mitarbeiter von „Kinder finden Natur in Lehre“ und 4 Tanzpädagogen aus ganz Deutschland ist es gelungen, unter der Leitung von [Künstlername] „Das Unmögliche möglich zu machen“. 260 SchülerInnen haben zu den Elementen (Feuer, Wasser, Erde, Luft) fächer-, klassen- und generationsübergreifend gearbeitet und dabei sämtliche Facetten des Lernens abgebildet. Die Verknüpfung von Bewegung, kultureller Bildung, naturwissenschaftlicher Arbeit und Fachunterricht ist beeindruckend gelungen. (2_SL2_028)

\footnotetext{
5 NGO: Non Governmental Organisation (Nichtregierungsorganisation).
} 


\section{2 ... an den Personen}

Eine weitere zentrale Eigenschaft der Facette zeigt sich über die verschiedenen Personen, an denen es nah dran zu sein gilt. Zeigt sich in Bezug auf das Schulumfeld und die Schulpartner noch die Vernetzung auf der organisationalen Mesoebene, so handelt es sich hier um die individuelle Mikroebene der täglichen Interaktionen, welche in dieser Eigenschaft bestimmend ist. Diese Personalität kann aufgrund der erhobenen Daten einerseits als Achtsamkeit im Sinne der (An-)Erkennung jedes Einzelnen an der Schule als einen Menschen, den es wahrzunehmen und zu würdigen gilt, verstanden werden. Gleichzeitig erscheint sie auch als eine wohlwollende, teilhabende Haltung. In der bewussten Zuwendung zu sich selbst und anderen findet das „Nah-dran-sein“ an der Person einen besonderen Ausdruck und weist Parallelen zum Konzept „Well-being“ auf. Bei unseren Erhebungen zeichnete sich dies sich vor allem durch die Präsenz von Schulleiter/innen im Gebäude aus.

Hierbei ist aber nicht ein „Patrouillieren“ im Schulhaus gemeint, sondern das Bemühen um die gezielte Kontaktaufnahme mit Lehrkräften und Schüler/innen. Schulleitungspersonen, die „,nah dran sind“, schaffen es durch Gespräche über aktuelle Geschehnisse und Herausforderungen der verschiedenen Gruppen bzw. Akteure Bescheid zu wissen, welche dadurch ein Gefühl der Anteilnahme und Teilhabe erfahren. Eine weitere Weise der Kontaktaufnahme sind Unterrichtsbesuche. Ziel kann die Evaluierung des Unterrichts (im Sinne des pädagogischen Konzepts der Schule) sein oder das Interesse sich ein Bild über die Arbeitsweisen der Schüler/innen zu machen, was in systematischer Form über einen Classroom Walkthrough (Schwarz 2013) erfolgen kann. Einen weiteren ,,nahen“ Einblick in das Schulgeschehen bieten Vertretungen. Allfällige Krankheitsfälle werden soweit möglich genützt, um selbst im Unterricht zu stehen und den Kontakt zu den Schüler/innen nicht zu verlieren. Einzelne Schulleiter/innen leben eine Politik der offenen Tür, um einerseits Präsenz zu zeigen, die andererseits aber auch die Möglichkeit für alle Beteiligten bietet, einzutreten und mit der Schulleitung in Kontakt zu treten.

Für den personalen Zugang von Schulleiter/innen finden sich in den Daten zahlreiche Belege, die von persönlichen Begegnungen im Sinne einer aktiven Beziehungsgestaltung bis hin zu personal verantwortetem pädagogischem und didaktischem Denken und Handeln zeugen. Die Handlungen der Schulleitung geraten dabei ins Spannungsfeld zwischen rechtlichen, politischen sowie wirtschaftlichen Vorgaben und personalen und pädagogischen Entscheidungen. Dabei ist sie in besonderem $\mathrm{Ma} ß$ gefordert, in diesem Spannungsfeld eine Balance herzustellen, wie das folgende Beispiel bei der Freistellung vom Dienst für den Besuch der Beerdigung eines Freundes nahelegt:

Aber ich darf keinen Lehrer beurlauben vom Dienst, wenn irgendein Freund vom Segelclub verstorben ist. Aber bin ich denn so bescheuert und beurlaub den nicht für eine Beerdigung? [...] Mein Gott, was passiert denn? Gar nichts. (1_SL1_043) 


\section{3 ... an der Forschung}

In einer weiterführenden Eigenschaft der Facette „Nah dran sein“ sind Forschung bzw. wissenschaftliche Erkenntnisse als Referenzrahmen für inhaltliche Argumentationen und die Weiterentwicklung der Schule von Bedeutung. Zahlreiche Daten weisen auf die Beschäftigung der Schulleiter/innen mit aktuellen Forschungsergebnissen zu schul- und bildungsrelevanten Themen und die Nutzung der erworbenen Kenntnisse als Grundlage für eigene Schul- und Unterrichtsentwicklungsprozesse oder zu deren Argumentation und Legitimation. Mehrere der untersuchten Schulen kooperieren mit diversen Bildungspartnern aus den Bereichen Forschung, Wirtschaft, Technik und Soziales zur Unterstützung regionaler Vernetzung und (Weiter-)Entwicklung eigener schulischer Konzepte. So finden sich an den untersuchten Schulen Kooperationen mit dem Max-Planck-Institut für Meteorologie oder diversen Universitäten, wobei Schulen sich einerseits für Forschungsprojekte offen zeigen und somit Institutionen Raum geben, um ihre aktuellen Forschungsergebnisse in den Unterricht und die Schule einfließen zu lassen bzw. diese in Zusammenarbeit zu entwickeln.

Die Schulleiter/innen lassen sich von Vorträgen und Veröffentlichungen aus verschiedenen Bereichen der Wissenschaft wie beispielsweise der Pädagogik, Neurobiologie oder Psychologie inspirieren und leiten. Eine Schule führte beispielsweise für Oberstufenschüler ein Gleitzeitmodell ein, um den Jugendlichen freizustellen, erst um 9:00 Uhr mit dem Unterricht zu beginnen. Anlass für diesen Entwicklungsschritt war die Auseinandersetzung mit neurobiologischen Erkenntnissen aus der Schlafforschung: Durch die Veränderung der Unterrichtszeiten soll die für die Verknüpfung und Vernetzung von erworbenem Wissen wertvolle zweite REM-Schlafphase, die laut neurowissenschaftlichen Erkenntnissen im Jugendalter zwischen 7:00 und 8:00 morgens stattfinden soll, nicht aufgrund eines frühen Unterrichtsbeginns durchbrochen werden.

Eine weitere Art des Nah-dran-seins über Kooperation stellen wissenschaftliche Begleitungen dar, die an einigen Schulen durchgeführt werden und meist einen evaluierenden Charakter haben.

Zum forschenden Lernen fand eine wissenschaftliche Untersuchung der Universität [Name] statt, die hohe Werte im Bereich der Selbstwirksamkeit, Kompetenzerleben, Autonomie und Eingebundenheit und die Gemeinschaft bei unseren Unterstufen- und MittelstufenschülerInnen (7. und 9. Jg.) ergab. (10_BU_013)

In den Interviews wird ein Mehrwert attestiert, der durch die Hereinnahme von Wissenschafter/innen dadurch erreicht wird, indem eingeschlagene Wege und Konzepte überprüft werden. Dies gibt den Akteuren an Schulen Hinweise und Sicherheit in Bezug auf ihr Handeln, was für die Weiterentwicklung der Schule wesentlich ist. Dabei kann die Perspektive der Wissenschaftler/innen Implizites explizit und damit für die Schule erst bearbeitbar machen. 


\section{4 ... am pädagogischen Gesamtkonzept}

In den vorhandenen Daten wird vielfach eine gelebte Haltung der Schulleitung deutlich sichtbar, die sich im Rahmen des jeweiligen Schulkontexts handlungsleitenden, pädagogischen Grundüberzeugungen verpflichtet fühlt. Diese sind dadurch gekennzeichnet, dass sie (a) am und zum Kind orientiert sind, (b) bei der Personalauswahl mitentscheidend sind, (c) die Bedürfnisse Aller berücksichtigen und sich in der beständigen Weiterentwicklung der Schule widerspiegeln. Sie zeigen, wie nah die Schulleitungen am bzw. hinter dem jeweiligen Schulprogramm bzw. den Schulkonzepten stehen, was sie jederzeit selbstbewusst, klar und deutlich vertreten. Des Weiteren legt die Auswertung der Daten nahe, dass Schulleiter/innen an den untersuchten Schulen eine große persönliche und starke inhaltliche Nähe zu den pädagogischen Inhalten und Grundkonzepten ihrer Schule haben. So vertreten sie nicht nur entschieden das Schulkonzept und Schulprogramm, sondern haben die Ausarbeitung an diesen für Schulen zentralen, programmatischen Dokumenten begleitet und entscheidend Einfluss genommen.

Die Schulleiter/innen sehen sich auch dafür verantwortlich, die wünschenswerte Qualität der Schule zu gewährleisten. Hierzu gehören Überprüfungen und Diskussionen des pädagogischen Konzepts und dessen Inhalte sowohl intern mit dem Kollegium, Eltern, Schüler/innen und weiteren Personen, die in unterschiedlicher Funktion in der Schule tätig sind, als auch mit externen Expert/innen, die beispielsweise Vorträge oder Workshops bei schulinternen Fortbildungen halten oder wissenschaftliche Untersuchungen durchführen. Das Einleiten von Entwicklungsprozessen kann als eine Kernaufgabe von erfolgreichem Schulleitungshandeln gesehen werden, in dem etwa Besuche von und an anderen Schulen organisiert werden. Eine wesentliche Aufgabe sehen die Schulleitenden auch in der sorgfältigen Einarbeitung neuer Lehrkräfte in das Konzept und die Pädagogik der Schule.

Zugleich scheinen Schulleiter/innen erfolgreicher Schulen gut darüber aufgeklärt zu sein, welche Auswirkungen minimale Änderungen auf das Gesamtkonzept haben können. Gerade deshalb sind auch sie es, die beispielsweise das pädagogische Konzept intern wie extern selbstbewusst und meinungsstark vertreten und den, wie es eine Schulleitung im Interview nennt, ,pädagogischen Goldschatz“ hüten:

Also sie hat von Anfang an eine Vision gehabt von Schule und die hat sie echt bis zuletzt so durchgehalten [...] und verfolgt, obwohl sie unglaubliche Anfeindungen auch erfahren hatte und auch ganz viel Protest, und trotzdem ist sie drangeblieben, das finde ich sehr beeindruckend [...] aber sie ist auch mutig und macht das dann und scheut nicht die Führung [...] sie ist sehr zielgerichtet und sie übernimmt Verantwortung und die Führung auch. (17_FGL_027)

\section{Diskussion und Ausblick}

Im internationalen Diskurs über die Wirksamkeit von Schulen haben sich Forschungsanliegen in den letzten Jahren verstärkt auf die Rolle der Schulleitung fokussiert, vor allem im Hinblick auf deren Einfluss auf die Entwicklung von Schule und 
Unterricht. Diesem Anliegen stellt sich das diesem Beitrag zugrundeliegende Forschungsprojekt, wenn es das Führungshandeln an 28 mit dem Deutschen Schulpreis ausgezeichneten Schulen untersucht. Die Ergebnisse daraus können weiterführende Erkenntnisse zur Qualitätsentwicklung von Schule und Unterricht generieren und in der Qualifizierung und Professionalisierung von Schulleiter/innen Anwendung finden. Über die phänomenologisch orientierte Herangehensweise wurde versucht, jene Wesensmerkmale aufzuspüren, die aus der Wahrnehmung der Akteure dem Handlungsgeschehen schulischer Alltagserfahrungen an den untersuchten Schulen zugrunde liegen. Dazu wurden die im Grounded Theory-Verfahren herausgearbeiteten Facetten der vielseitigen Aktivitäten von Schulleiter/innen, die sich in den Aussagen im Feld zeigten, in Form eines Antwortregisters mehrperspektivisch zusammengefasst.

Die im Register versammelten Antworten sind Ausdruck der vielen Facetten, wie sich Schulleitungshandeln den Akteuren an den besuchten Schulen zeigt. Sie weisen im Einzelnen auf je eine Erscheinungsform von Führung, sind aber immer auch mit anderen verbunden. Daher lassen sich die gewonnenen Facetten nicht isoliert betrachten, sondern nur in ihrer Vernetzung verstehen. Sie sind nicht in „Wirkungsketten“ monokausaler Zusammenhänge (z.B. Einsatz von Führungsstilen) zu denken, sondern als „Wirkungsnetze“ (Dörner 1983, S. 23), da sich einzelne Facetten von Führungshandeln in unterschiedlichen Akteurskonstellationen auf verschiedene Weise verbinden. In diesem Sinne verstehen sich die einzelnen Facetten als wesentliche Bestimmungsstücke eines Facettenmodells, als Teile eines größeren Ganzen. Erst im Zusammenspiel der unterschiedlichen Facetten lässt sich jenes Führungshandeln an ausgezeichneten Schulen erklären, das der Komplexität schulischer Handlungszusammenhänge gerecht werden kann.

Im gegenständlichen Forschungsprojekt wurden inzwischen 55 Facetten erfolgreichen Führungshandelns registriert, die sich in vielfältige Eigenschaften und Ausprägungen auffächern lassen, was sich in unterschiedlichen Beziehungen und Verhältnissen zeigt: Die Facette ,ausbalancieren“ ist, wie die im Beitrag vorgestellte „nah dran sein“, relational bestimmt, die Facette „öffnen von Türen“ hat einen räumlichen Bezug, weist zugleich aber auch auf die Zukunft, die sich perspektivisch eröffnet. Ähnlich enthält die Facette „Möglichkeitsräume schaffen“ eine räumliche und zeitliche Perspektive. Daraus wird in der Folge ein Facettenmodell entwickelt, das der Vielschichtigkeit schulischen Entwicklungsgeschehens gerecht werden soll, in das Führungshandeln eingebettet ist. Denn Schule und Unterricht - ,jeweils gedacht als System - sind definiert durch einen vielfältigen Umgang mit beteiligten Bestimmungsstücken, deren Vernetztheit, Beziehungen, Verbindungen und Verknüpfungen zu einem dynamischen Geschehen mit vielen, oft auch widersprüchlichen Zusammenhängen führen“ (Wiesner und Schreiner 2019, S. 127). Über die Vernetzung der Facetten lässt sich aufzeigen, in welch unterschiedlichen Formationen und Ausprägungen sich das Wahrnehmen von Führung in gegenseitigen Beeinflussungsverhältnissen bewähren muss. Eine Systematisierung dazu steht noch aus.

Das entstehende Antwortregister, dessen Grundstruktur in diesem Beitrag beispielhaft aufgezeigt wurde, kann über das ausdifferenzierte Facettenmodell die Grundlage für die Analyse der Ausprägung dynamischer Eigenschaften von schulischen Führungskräften dienen und damit Anregungen zur individuellen Weiterent- 
wicklung geben. So kann deutlich werden, dass Führung sich nicht über die Position definiert, sondern als Aktion wirksam wird, geteilte Verantwortung voraussetzt und erst in gemeinsamem Tun des jeweiligen Kontexts seine Wirkmacht erhält. Für die Qualifizierung und Professionalisierung von Schulleiter/innen kann es darüber hinaus die Kernelemente zur Erstellung von Aus- und Weiterbildungscurricula liefern, in denen die unterschiedlichen Facetten erfolgreichen Führungshandelns im Sinne eines größeren Ganzen aufeinander bezogen werden. Im Bereich wissenschaftlicher Forschung lässt sich das Antwortregister über Folgeuntersuchungen - etwa unter Einbezug weiterer Akteure - anreichern, um damit die Gesamtschau von neuen Erkenntnissen zum schulischen Führungshandeln zu erweitern. Hierzu gilt es forschungsmethodische Zugänge zu finden, die das dynamische Geschehen gelebter Führungspraxis zu erfassen vermögen.

Funding Open access funding provided by University of Innsbruck and Medical University of Innsbruck.

Open Access Dieser Artikel wird unter der Creative Commons Namensnennung 4.0 International Lizenz (http://creativecommons.org/licenses/by/4.0/deed.de) veröffentlicht, welche die Nutzung, Vervielfältigung, Bearbeitung, Verbreitung und Wiedergabe in jeglichem Medium und Format erlaubt, sofern Sie den/die ursprünglichen Autor(en) und die Quelle ordnungsgemäß nennen, einen Link zur Creative Commons Lizenz beifügen und angeben, ob Änderungen vorgenommen wurden.

\section{Literatur}

Albers, A. (2016). Schulwettbewerbe als Impuls für Schulentwicklung. Wiesbaden: Springer. Research Altrichter, H., \& Maag Merki, K. (Hrsg.). (2010). Handbuch Neue Steuerung im Schulsystem. Wiesbaden: Springer VS.

Altrichter, H., Helm, C., \& Kanape-Willingshofer, A. (2012). Unterrichts- und Schulqualität. http://www. sqa.at/pluginfile.php/988/coursecat/description/qualitaet_von_unterricht_und_schule.pdf. Zugegriffen: 27. Apr. 2019.

Ammann, M. (2018). Leadership for learning as experience: introducing the use of vignettes for research on leadership experiences in schools. International Journal of Qualitative Methods, 17(1), 1-13. https://doi.org/10.1177/1609406918816409.

Bell, L., Bolam, R., \& Cubillo, L. (2003). A systematic review of the impact of school head teachers and principals on student outcomes. London: Institute of Education.

Beutel, S.-I., Höhmann, K., Pant, H. A., \& Schratz, M. (Hrsg.). (2016). Qualitätsbereiche für zukunftsweisende Praxis. Handbuch Gute Schule, Bd. 6. Seelze: Kallmeyer.

Biesta, G. (2013). The beautiful risk of education. Boulder, London: Paradigm Publishers.

Biesta, G. (2017). The Rediscouvery of teaching. New York, London: Routledge.

Brüchle, J., Düvel, A., Keil, T., \& Wenzelewski, C. (2015). Eine Analyse mikropolitischer Einflusstaktiken von Führungskräften in der ersten disziplinarischen Führungsposition zur Interessendurchsetzung gegenüber dem eigenen Team. In A. Mucha, A. Endemann \& D. Rastetter (Hrsg.), Mikropolitik am Arbeitsplatz. Qualitative Studien zur Anwendung von Taktiken in Untenehmen (S. 139-167). München, Mering: Rainer Hampp.

Cappella, E., Aber, J.L., \& Kim, H. Y. (2016). Teaching beyond achievement tests: perspectives from developmental and education science. In D. H. Gitomer \& C. A. Bell (Hrsg.), Handbook of research on teaching (S. 249-347). Washington, DC: American Educational Research Association.

Damon, W. (2009). The path to purpose: How young people find their calling in life. New York: Free Press.

Day, C., Sammons, P., Hopkins, D., Harris, A., Leithwood, K., Gu, Q., \& Brown, E. (2010). 10 strong claims about successful leadership. Nottingham: National College for Teaching and Leadership.

Dellwing, M., \& Prus, R. (2012). Einführung in die interaktionistische Ethnografie. Soziologie im Aussendienst. Wiesbaden: Springer VS.

Dörner, D. (1983). Die Anforderungen komplexer und unbestimmter Probleme. In D. Dörner, H. W. Kreuzig, F. Reither \& T. Stäudel (Hrsg.), Vom Umgang mit Unbestimmtheit und Komplexität (S. 19-104). Bern: Huber.

Duden (2001). Band 5. Das Fremdwörterbuch. Mannheim, Leipzig, Wien, Zürich: Dudenverlag. 
Ecclestone, K. (2007). Resisting images of the 'diminished self': the implications of emotional well-being and emotional engagement in education policy. Journal of Education Policy, 22(4), 455-470.

Fauser, P., Prenzel, M., \& Schratz, M. (Hrsg.). (2007). Was für Schulen! Gute Schule in Deutschland: Der Deutsche Schulpreis 2006. Seelze: Klett / Kallmeyer.

Flick, U. (2008). Triangulation - Eine Einführung. Wiesbaden: VS.

Gadamer, H.-G. (1963). Die phänomenologische Bewegung. Philosophische Rundschau, 11(1/2), 1-45.

Hallinger, P. (2009). Leadership for 21st century schools: from instructional leadership to leadership for learning. Hong Kong: The Hong Kong Institute of Education.

Hallinger, P., \& Heck, R. H. (2011). Conceptual and methodological issues in studying school leadership effects as a reciprocal process. School Effectiveness and School Improvement, 22(2), 149-173.

Harazd, B. (2010). Schulleitungstypen in eigenverantwortlichen Schulen und ihr Zusammenhang zur Schulqualität. In N. Berkemeyer, et al. (Hrsg.), Jahrbuch der Schulentwicklung. Band 16 (S. 261-288). Weinheim: Juventa.

Hargreaves, A., \& Shirley, D. (2009). The fourth way: The inspiring future for educational change. Thousand Oaks: Corwin Press.

Hargreaves, A., \& Shirley, D. (2012). The global fourth way: The quest for educational excellence. Thousand Oaks: Corwin Press.

Heck, R.H., \& Hallinger, P. (2010). Collaborative leadership effects on school improvement: integrating unidirectional- and reciprocal effect models. The Elementary School Journal, 111(2), 226-252.

Helliwell, J., Layard, R., \& Sachs, J. (Hrsg.). (2016). World happiness report 2016 update: volume I. https://worldhappiness.report/ed/2016/. Zugegriffen: 27. Apr. 2019.

Helsper, W. (2002). Lehrerprofessionalität als antinomische Handlungsstruktur. In M. Kraul, W. Marotzki \& C. Schweppe (Hrsg.), Biographie und Profession (S. 64-102). Bad Heilbrunn: Klinkhardt.

Helsper, W. (2004). Antinomien, Widersprüche, Paradoxien: Lehrerarbeit - ein unmögliches Geschäft? Eine strukturtheoretisch-rekonstruktive Perspektive auf das Lehrerhandeln. In B. Koch-Priewe, F.-U. Kolbe \& J. Wildt (Hrsg.), Grundlagenforschung und mikrodidaktische Reformansätze zur Lehrerbildung (S. 49-98). Bad Heilbrunn: Klinkhardt.

Husserl, E. (1962). Die Krisis der europäischen Wissenschaften und transzendentale Phänomenologie. Husserliana VI. Den Haag: Martinus Nijhoff.

Husserl, E. (1990). Die phänomenologische Methode. Ausgewählte Texte/Edmund Husserl, Bd. 1. Stuttgart: Reclam.

Klein, D. (2016). Instructional Leadership in den USA: Ein Erfolgsmodell für Deutschland? Tertium Comparationis, 22(2), 203-230.

Kohlstock, B., \& Heinrich, M. (2015). Ambivalenzen des Ökonomischen. Analysen zur ,Neuen Steuerung ‘ im Bildungssystem. Wiesbaden: Springer.

Kron, F. W. (1999). Wissenschaftstheorie für Pädagogen. München: Reinhardt.

Kruse, J. (2014). Qualitative Interviewforschung. Ein integrativer Ansatz. Weinheim, Basel: Beltz Juventa. Lakoff, G., \& Johnson, M. (2001). Metaphors we live by. Chicago, London: University of Chicago Press. Lamnek, S. (2005). Qualitative Sozialforschung. Lehrbuch. Weinheim, Basel: Beltz.

Leithwood, K., \& Seashore Louis, K. (2012). Linking leadership to student learning. San Francisco: Wiley. Leithwood, K., Louis, K.S., Anderson, S., \& Wahlstrom, K. (2004). How leadership influences student learning: A review of research for the learning from leadership project. New York: The Wallace Foundation.

Leithwood, K., Louis, K. S., Anderson, S., Wahlstrom, K., Anderson, S., Mascall, B., \& Gordon, M. (2009). How successful leadership influences student learning: the second installment of a longer story. In A. Hargreaves, A. Lieberman, M. Fullan \& D. Hopkins (Hrsg.), Second handbook of educational change (S. 611-629). Dordrecht: Springer.

Messner, R. (2003). PISA und Allgemeinbildung. Zeitschrift für Pädagogik, 48(3), 400-412.

Meyer-Drawe, K. (2017). Phenomenology as a philosophy of experience-Implications for pedagogy. In M. Ammann, T. Westfall-Greiter \& M. Schratz (Hrsg.), Erfahrungen deuten - Deutungen erfahren. Experiential vignettes and anecdotes as research, evaluation and mentoring tool (S. 13-21). Innsbruck: StudienVerlag.

OECD (2017). How's life? 2017: measuring well-being. Paris: OECD Publishing.

Ontario (2016). Ontario's well-being strategy for education: discussion document. Toronto: Ministry of Education.

Oswald, H. (2003). Was heißt qualitativ forschen? In B. Friebertshäuser \& A. Prengel (Hrsg.), Handbuch Qualitative Forschungsmethoden in der Erziehungswissenschaft (S. 71-87). Weinheim, München: Juventa. 
Pietsch, M., Lücken, M., Thonke, F., Klitsche, S., \& Musekamp, F. (2016). Der Zusammenhang von Schulleitungshandeln, Unterrichtsgestaltung und Lernerfolg. Zeitschrift für Erziehungswissenschaft, 19, $527-555$.

Reh, S. (2012). Beobachtungen aufschreiben. In H. de Boer \& S. Reh (Hrsg.), Beobachtung in der Schule - Beobachten lernen (S. 115-130). Wiesbaden: Springer VS.

Rosenthal, G. (2014). Interpretative Sozialforschung. Eine Einführung (4. Aufl.). Weinheim, Basel: Beltz Juventa.

Salokangas, M., \& Ainscow, M. (2018). Inside the autonomous school. Making sense of a global educational trend. London, New York: Routledge.

Schratz, M. (2017). Innovative Schulen - exzellente Praxis. Tendenzen der Schulentwicklung am Beispiel des Deutschen Schulpreises. In U. Steffens, K. Maag Merki \& H. Fend (Hrsg.), Schulgestaltung. Aktuelle Befunde und Perspektiven der Schulqualitäts- und Schulentwicklungsforschung (S. 231-247). Münster: Waxmann.

Schratz, M., \& Hartmann, M. (2009). Schulautonomie in Österreich: Bilanz und Perspektiven für eine eigenverantwortliche Schule. In W. Specht (Hrsg.), Nationaler Bildungsbericht Österreich (Bd. 2, S. 323-340). Graz: Leykam.

Schratz, M., Schwarz, J.F., \& Westfall-Greiter, T. (2012). Lernen als bildende Erfahrung: Vignetten in der Praxisforschung. Innsbruck: Studien Verlag.

Schratz, M., Wiesner, C., Rößler, L., Schildkamp, K., George, A. C., Hofbauer, C., \& Pant, H. A. (2019). Möglichkeiten und Grenzen evidenzorientierter Schulentwicklung. In S. Breit, F. Eder, K. Krainer, C. Schreiner, A. Seel \& C. Spiel (Hrsg.), Nationaler Bildungsbericht Österreich 2018: Fokussierte Analysen und Zukunftsperspektiven für das Bildungswesen (S. 403-453). Graz: Leykam. https://doi. org/10.17888/NBB2018-2-10.

Schwarz, J. (2013). Unterrichtsbezogene Führung durch “Classroom Walkthrough”: Schulleitungen als Instructional Leaders. Innsbruck: StudienVerlag.

Schwarz, A., \& Brauckmann, S. (2015). Between facts and perception: The area close to schools as a context factor in leadership. Schumpeter Discussion Papers. Wuppertal: University of Wuppertal.

Shirley, D. (2016). Das Gebot für eine Neugestaltung von Bildung. In S.-I. Beutel, K. Höhmann, H. A. Pant \& M. Schratz (Hrsg.), Qualitätsbereiche für zukunftsweisende Praxis. Handbuch Gute Schule, (Bd. 6, S. 8-11). Seelze: Kallmeyer.

Shirley, D. (2017). The new imperatives of educational change. Achievement with integrity. New York: Routledge.

Spratt, J. (2019). Wellbeing, equity and education: a critical analysis of policy discourses of wellbeing in schools. Cham: Springer.

SQA (2018). SQA Evaluation 2018 - Ergebnisse der quantitativen Erhebung. http://www.sqa.at/. Zugegriffen: 27. Apr. 2019.

Strauss, A., \& Corbin, J. (1996). Grundlagen qualitativer Sozialforschung. Weinheim: Beltz.

Strübing, J. (2008). Grounded theory. Wiesbaden: VS.

Townsend, T., \& MacBeath, J. (Hrsg.). (2011a). International handbook of leadership for learning, Part one. Dordrecht: Springer.

Townsend, T., \& MacBeath, J. (Hrsg.). (2011b). International handbook of leadership for learning, Part two. Dordrecht: Springer.

Waldenfels, B. (2007). Antwortregister. Frankfurt, M.: Suhrkamp.

Wiesner, C., \& Schreiner, C. (2019). Implementation, Transfer, Progression und Transformation: Vom Wandeln von Routinen zur Entwicklung von Idfentität. Von Interventionen zu Innovationen, die bewegen: Bausteine für ein Modell zur Schulentwicklung durch Evidenz(en). In C. Schreiner, C. Wiesner, S. Breit, P. Dobbelstein, M. Heinrich \& U. Steffens (Hrsg.), Praxistransfer Schul- und Unterrichtsentwicklung (S. 79-139). Münster: Waxmann.

Wiesner, C., George, A.-C., Rauscher, E., Schratz, M., \& Kemethofer, D. (2016). Schulleitung im Wandel: Neue Herausforderungen für SchulleiterInnen. SchulVerwaltung aktuell, 4, 100-102.

Wolff, S. (2005). Dokumenten- und Aktenanalyse. In U. Flick, E. von Kardorff \& I. Steinke (Hrsg.), Qualitative Forschung. Ein Handbuch (S. 502-513). Hamburg: Rowohlt. 